\title{
Effects of thymoquoinone on obese rats liver
}

\author{
Işınsu Aydın*, Elfide Gizem Kıvrak, Hande Tüfek, Gülay Yıldız, Berrin Zuhal Altunkaynak, Süleyman Kaplan
}

Department of Histology and Embryology, Faculty of Medicine, Ondokuz Mayis University, Samsun, Turkey

\section{ARTICLE INFO}

\section{* Correspondence to:}

Işınsu Aydin

Department of Histology and Embryology, Faculty of Medicine,

Ondokuz Mayıs University, Samsun, Turkey e-mail: isinsuaydin@gmail.com

\section{Keywords:}

Liver

Obesity

Rat

Stereology

Thymoquoinone

\section{ABSTRACT}

Obesity is a growing and epidemic problem in modern societies due to the intensive llifestyle, high carbohydrate or fat dietary intake, and reduced energy consumption. Nigella sacra is an annual herbaceous plant of the Ranunculaceae family and Thymoquoinone (TQ) is the main component of Nigella sativa. TQ possess anti-diabetic, anti-oxidant, hepatoprotective, neuroprotective, nephroprotective, anti-tumor, anti-mutagenic and TQ is prevent the membrane lipid peroxidation in hepatocytes. In this study, we aimed to detect effects of high fat diet induced obesity and TQ on liver by using physical disector and Cavalieri methods. This study was including four groups: Non-obese control, obese control, non-obese TQ and obese-TQ groups. Liver of rats of all groups were processed and staning with hematoksilin eosin for stereological and light microscopic analysis. The volume of liver was estimated by Cavalieri methods and the number of hepatocyte was investigated by physical dissector. The numerical densities of hepatocytes for both the obese-control were significantly decreased in comparison to the control group. But TQ treatment lead to a significant increase of hepatocyte number in obese TQ group after fatty diet induced obesity. Besides, there weren't significantly differences about the total number of hepatocite between the nonobese-control group and nonobese TQ group. The light microscopical images showed steatosis, ballooning degeneration and many hypertrophied cells in the obese control group. We observed healthy structure and a hepatocyte cords, central veins and portal triads in light microscopical images of the other groups. This study provided information that TQ has a protective effect on the liver in terms of liver volumes, number of hepatocytes and histological structure in obese rat model.

J. Exp.Clin.Med., 2014; 31:135 